\title{
Tomographic image quality of rotating slat versus parallel hole collimated SPECT
}

\author{
Roel Van Holen ${ }^{1}$, Steven Staelens ${ }^{1,2}$, Stefaan Vandenberghe ${ }^{1}$ \\ ${ }^{1}$ ELIS Department, MEDISIP, IBBT, Ghent University, IBBT, IBiTech, Ghent, \\ Belgium \\ ${ }^{2}$ Molecular Imaging Center Antwerp, Faculty of Medicine, Antwerp University, \\ Antwerp, Belgium \\ E-mail: Roel.VanHolen@UGent.be
}

\begin{abstract}
Parallel and converging hole collimators are most frequently used in nuclear medicine. Less common is the use of rotating slat collimators for Single Photon Emission Computed Tomography (SPECT). The higher photon collection efficiency, inherent to the geometry of rotating slat collimators results in much lower noise in the data. However, plane integrals contain spatial information in only one direction, whereas line integrals provide two-dimensional information. It is not a trivial question whether the initial gain in efficiency will compensate for the lower information content in the plane integrals. Therefore, a comparison of the performance of parallel hole and rotating slat collimation is needed. This study compares SPECT with rotating slat and parallel hole collimation in combination with MLEM reconstruction with accurate system modeling and correction for scatter and attenuation. A contrast-to-noise study revealed an improvement of a factor 2 to 3 for hot lesions and more than a factor of 4 for cold lesion. Furthermore, a clinically relevant case of heart lesion detection is simulated for rotating slat and parallel hole collimators. In this case, rotating slat collimators outperform the traditional parallel hole collimators. We conclude that rotating slat collimators are a valuable alternative for parallel hole collimators.
\end{abstract}

\section{Introduction}

Single Photon Emission Computed Tomography (SPECT) relies on the detection of gamma rays that result from the decay of radioisotopes, administered to the patient. Unlike optical photons, high energy photons or gamma rays can not be focussed by traditional lenses. In order to extract directional information from isotropically emitted gamma rays, collimators are used instead. Their task is to absorb all photons, not traveling according to the collimator-imposed direction. As a result, only photons traveling in the required direction will pass the collimator. A major drawback is that only a limited amount of photons reach the detector. This low sensitivity typically results in noisy SPECT images.

The most common collimator used in nuclear medicine is the parallel hole $(\mathrm{PH})$ collimator. It is constructed as a slab of highly attenuating material (lead or tungsten) 
with a numerous amount of closely packed long, parallel holes. A PH collimator makes direct one-to-one projection images of the source on the detector. Therefore, the FOV of a parallel hole collimator is equal to the size of the detector. This property makes this type of collimator generally applicable in clinical practice. In brain SPECT studies and sometimes in cardiac studies, the large FOV is not required. Focussing collimators are then used to obtain higher sensitivity and better spatial resolution. In fan-beam collimators the holes are tilted toward a focal line while cone-beam collimators have their holes focussed to a point. Because the focal line of a fan-beam collimator is parallel to the axis of rotation of the camera, we only have a magnification effect in the transaxial direction and no magnification in axial direction. On the other hand, projections made with a conebeam collimator are magnified both in axial and transaxial direction. Finally, pinhole and multi-pinhole collimators are often used, especially in pre-clinical SPECT systems. Pinhole-based systems can obtain very good spatial resolution and sensitivity at the cost of a decreased FOV. All the aforementioned types of collimation however suffer from the same trade-off between sensitivity, spatial resolution and FOV. For a discussion on the most common SPECT collimators with respect to these three criteria, the reader is referred to the excellent overview papers by Wieczorek (Wieczorek and Goedicke 2006), Accorsi (Accorsi 2006) and Moore (Moore et al 1992).

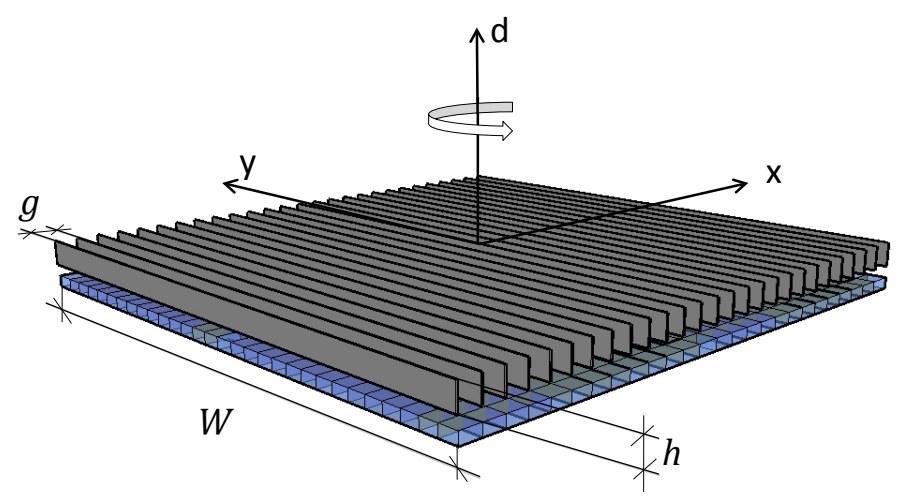

Figure 1. The geometry of a rotating slat collimator.

Rotating slat (RS) collimators (figure 1), which are the subject of this paper, break with the traditional collimators described above. They fundamentally differ in the sense that instead of line integrals, they measure plane integrals. Furthermore, rotating slat collimators exhibit a different resolution/sensitivity relationship (Vandenberghe et al 2006). RS collimation has been used in combination with solid state detectors in the SOLSTICE (SOLid STate Imager with Compact Electronics) design proposed by Gagnon (Gagnon et al 2001, Griesmer et al 2001). Due to the rotating slat design, only a limited number of detector elements is required to fill a strip area which reduces the cost. A similar design has been published by Entine (Entine et al 1979) about 20 years ago, combining a CdTe detector with a parallel plate collimator. Before this, the 
design of a linear detector has been proposed independently by Keyes (Keyes 1975) and Tosswill (Tosswill 1977). Traditional rectangular SPECT detectors have been studied in combination with rotating slat collimators by Webb, who found an increased sensitivity of about a factor of 40 for the rotating slat concept (Webb et al 1992). Due to the different nature of the data measured by this collimator, other reconstruction techniques have to be used to reconstruct images. Analytic reconstruction methods for planar and tomographic acquisitions have been derived by Lodge (Lodge et al 1995, Lodge et al 1996). A 3D iterative reconstruction algorithm for the SOLSTICE camera has been proposed by Wang (Wang et al 2004) and by Zeng (Zeng et al 2003).

A drawback of reconstructing plane integral data measured by a rotating slat collimator, to $3 \mathrm{D}$ images is the much longer computation time needed compared to a parallel hole reconstruction. This problem was solved in an earlier paper with the development of a reconstruction method that uses two updates per iteration, one in plane integral space and one in sinogram space (Van Holen et al 2007). Among different MLEM implementations to reconstruct plane integral data, this method was found to be the fastest. Furthermore, it maintains image quality.

Planar RS imaging with Filtered Back-Projection (FBP) reconstruction has previously been studied by Lodge (Lodge et al 1995). Their work shows an advantage in signal-tonoise ratio (SNR) over a PH collimator for sparse activity distributions and an enhanced contrast in small hot spots. A previous comparison of planar image quality, using accurate system modeling in an iterative reconstruction, indicates improved contrastto-noise ratios up to a factor 3, even in large objects approaching the size of the fieldof-view (FOV) of the camera (Van Holen et al 2008). In the tomographic case, FBP reconstructed SPECT images with a RS collimator again confirmed improved noise characteristics for small objects (smaller than $10 \mathrm{~cm}$ ) and showed improved contrast only for small regions of high tracer uptake (Lodge et al 1996). In (Wang et al 2004), next to better hot spot contrast, also an increased contrast was found for cold lesions. However, in this study, the SOLSTICE detector was used and the image quality improvement was not only due to collimation with slats but also due to the combined effect of small detector width, better collimator resolution and solid state detector material. Moreover, in this comparison, no model for depth-dependent detector blurring was used in the reconstruction. Finally, in (Zhou et al 2010), where analytical collimator models were used, a comparable performance of $\mathrm{PH}$ and $\mathrm{RS}$ collimation was found, depending on object size and object distance.

In this paper, realistic measurements are simulated and all detector and collimator parameters will be matched in order to make a comparison between RS and $\mathrm{PH}$ collimation. This also implies that an attenuating medium will be present and accurately modeled. Therefore, we first need to validate whether classical scatter and attenuation correction techniques can be applied to plane integral reconstruction. After the development of an appropriate attenuation correction technique for the tomographic image reconstruction of plane integral data, we will compare image quality to a parallel hole collimator with identical field-of-view by means of a contrast-to-noise analysis. 
Next, a realistic Tc-99m-MIBI scan will be simulated on both a SPECT scanner equipped with $\mathrm{RS}$ and $\mathrm{PH}$ collimators to demonstrate the use of slat collimation in clinical practice. 


\section{Materials and Methods}

\subsection{Monte Carlo simulation models}

Geant4 Application for Tomographic Emission (GATE) (Santin et al 2003) was used to simulate the rotating slat and the parallel hole acquisitions. The detector was identical for both systems and was modeled as a pixelated solid state detector consisting of $192 \times 192$ individual CdZnTe (CZT) pixels of $1.8 \mathrm{~mm} \times 1.8 \mathrm{~mm}$. The thickness of the CZT was set to $5 \mathrm{~mm}$. The energy resolution of the CZT was set to $5 \%$ at $140 \mathrm{keV}$. To make the efficiency of the detector independent of the collimator type, the active area of one pixel was set to be only $1.5 \mathrm{~mm} \times 1.5 \mathrm{~mm}$. With $g=1.5 \mathrm{~mm}$, the collimator septa have a width of $0.3 \mathrm{~mm}$ thickness.

The RS collimator of figure 1 was simulated as 193 parallel lead slats of height $h=40 \mathrm{~mm}$, placed in between two neigbouring pixel rows. The thickness of a slat was set to $0.3 \mathrm{~mm}$ while the length was equal to the length $W$ of the detector $(W=345.6 \mathrm{~mm})$. The PH collimator had the same height and thickness as the RS collimator and was matched to the pixelated detector. This resulted in a parallel hole collimator with square holes of $1.5 \mathrm{~mm} \times 1.5 \mathrm{~mm}$. The collimator resolution is thus matched for both collimators and is $5 \mathrm{~mm}$ at $10 \mathrm{~cm}$ collimator distance. The RS collimator/detector

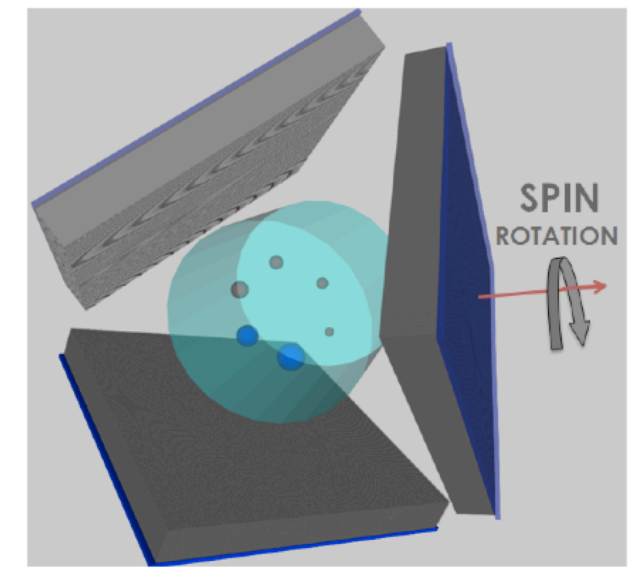

(a)

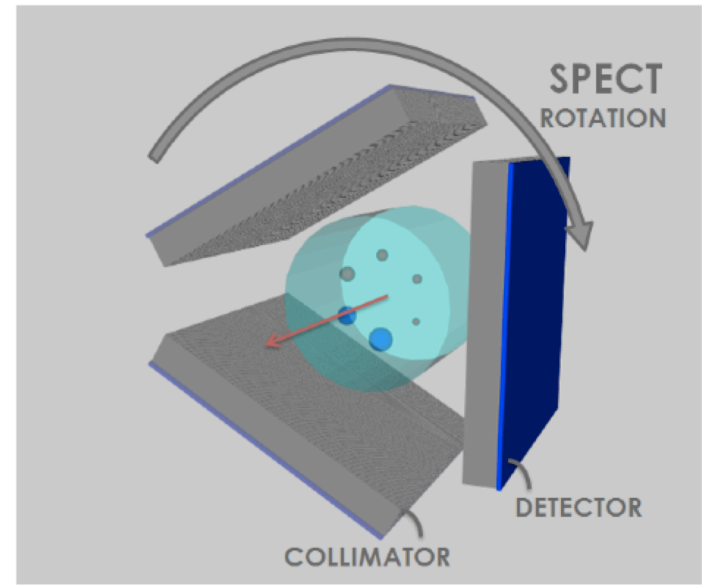

(b)

Figure 2. The geometry of the simulated SPECT camera equipped with (a) a RS collimator and (b) a PH collimator. Next to the SPECT rotation, a RS acquisition also needs a spin rotation of each detector.

pair was rotated around in a spin-rotation in 90 discrete steps (figure 2(a)). The PH collimator/detector pair did not rotate around its orthogonal axis during simulation. For both the RS and PH simulations, the collimator/detector pair was rotated around the scanner's central axis (figure $2(\mathrm{~b})$ ) in 90 discrete steps over $360^{\circ}$. Figure 2 (a) shows the three headed rotating slat camera that was modeled while in figure 2 (b) the three headed parallel hole system is shown. Particle interactions that are simulated 
include photo-electric effect, Compton and Raleigh scattering, electron ionization and Bremsstrahlung.

\subsection{Scatter estimation}

The Dual Energy Window (DEW) scatter estimation technique (Koral et al 1990) was used to correct for scatter in both the data acquired with a RS collimator and the PH collimator. According to this method, the scatter estimate is calculated as follows:

$$
g_{E S T}=k\left(g_{S W} \frac{w_{M W}}{w_{S W}}\right)
$$

with $g_{S W}$ the data measured in the scatter window. Since Tc-99m will be used throughout this study, the width of our main energy window $w_{M W}$ was chosen $14 \mathrm{keV}$ around $140 \mathrm{keV}$ and $w_{S W}$, the width of the scatter window was chosen $10 \mathrm{keV}$ and was located around $125 \mathrm{keV}$.

To investigate whether the DEW scatter correction technique will also be valid for RS collimation, two cylindrical phantoms filled with Tc-99m in water, were simulated in GATE. Their respective diameters were $20 \mathrm{~cm}$ and $35 \mathrm{~cm}$, while their height was fixed at $20 \mathrm{~cm}$. The rotation radius (distance from center of rotation to collimator) was $17.5 \mathrm{~cm}$ and $22.5 \mathrm{~cm}$ respectively. The respective activities were $3.7 \mathrm{MBq}$ and $11.3 \mathrm{MBq}$ while 13.5 minutes of acquisition time were simulated. Both cylinders were placed in the center of the field of view and aligned with the scanner's central axis.

Thanks to the history tracking of the detected events, GATE flags every detection that scattered in the phantom. Consequently, the true scatter distribution $g_{T R U E}$ is known. In order to study the feasibility of a spectral based scatter estimation correction, we looked at the energy spectra of respectively PH collimated data and RS collimated data. For both the small and large cylinder, detections originating from a spherical region with $10 \mathrm{~cm}$ diameter in the center of the phantom were selected to generate an energy spectrum. To study the spatial dependency of the spectrum also detections originating from a spherical region at a peripheral position, offset from the center by $10 \mathrm{~cm}$ in both transaxial dimensions and by $5 \mathrm{~cm}$ along the scanner's central axis, were selected in the large cylinder to generate a third energy spectrum. For comparison, the three spectra were generated for both $\mathrm{PH}$ and RS. As a quantitative validation, the estimated scatter fraction was compared to the true scatter fraction. No attempt is made to make a validation of spatial distributions at the level of the projections.

\subsection{Attenuation correction}

2.3.1. Implementation Since the data are affected by attenuation, we need a method to compensate for the loss of photons at larger depths. An attenuation correction method which fully models the attenuation along each possible ray of projection as used by Zeng et al. (Zeng et al 1991) is used for the reconstruction of the PH data. However, for RS data, this method is only possible in a fully 3D reconstruction algorithm which directly maps image to the plane integrals using one single system matrix. Since our 


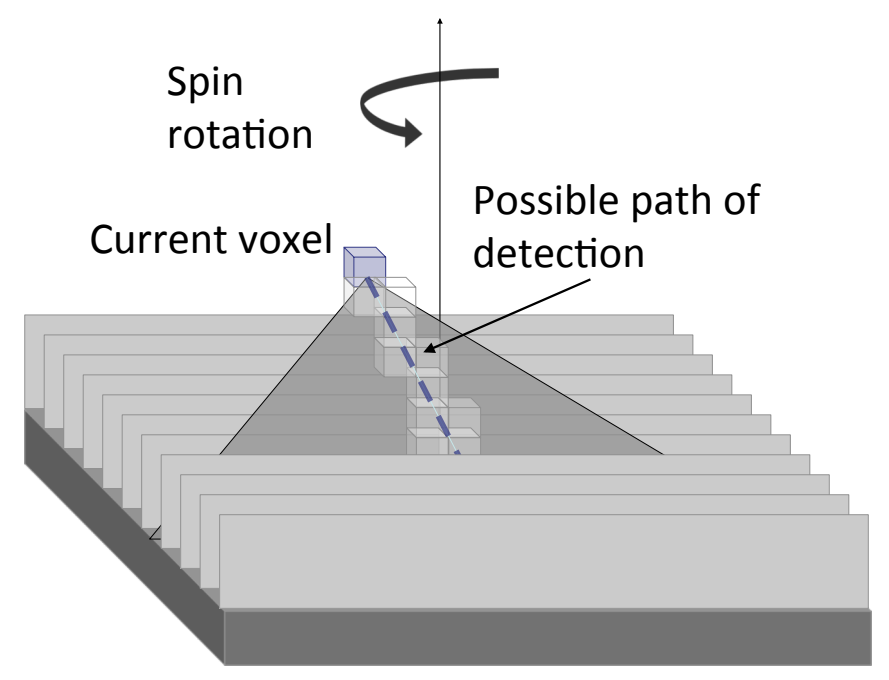

(a)

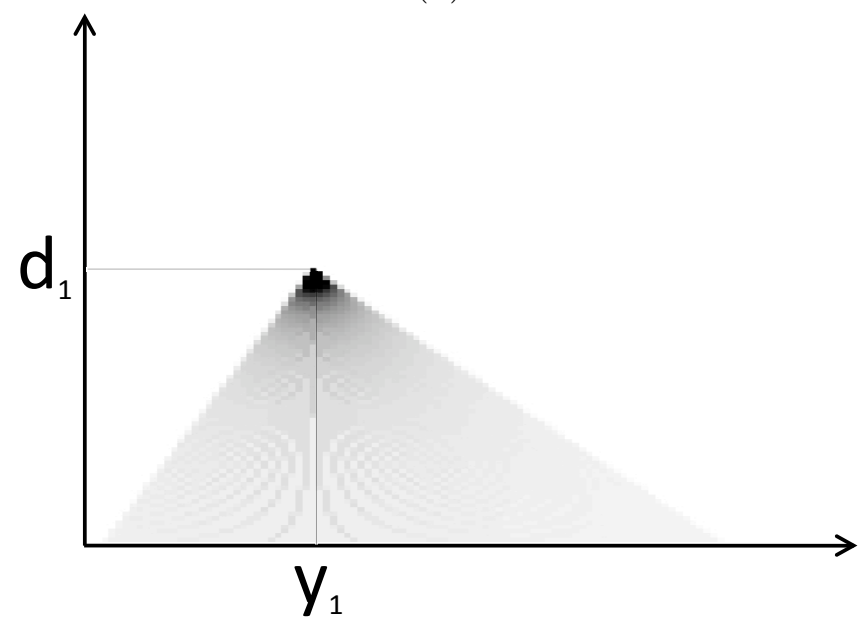

(b)

Figure 3. (a) One of the possible paths along which attenuation has to be calculated. This calculation needs to be repeated for every path in the fan, for every voxel, every spin and every SPECT angle. (b) The sensitivity weighted intersection lengths for all possible paths of detection for one voxel at one spin angle.

reconstruction method splits the system matrix in two separate ones, the original paths of detection are lost and this method can not be applied. Furthermore, tracing every possible ray for every spin and SPECT angle would be too tedious. Therefore, we base ourselves on Chang's attenuation correction method (Chang 1978) which calculates an average attenuation factor at each voxel. However, where Chang calculates the mean attenuation value over all SPECT angles for every voxel, our method will calculate an average attenuation coefficient $c$ over all spin angles $\phi$ for every voxel $(x, y, z)$ and for 
every SPECT angle $\theta$ :

$$
c(x, y, z, \theta)=\left(\frac{1}{\Phi} \sum_{i=0}^{\Phi} \exp \left(-(\mathrm{M} L)_{\phi_{i}}\right)^{-1},\right.
$$

with $(\mathrm{M} L)_{\phi_{i}}$ the mean attenuation-length product for spin angle $\phi_{i}$. The calculation of $(\mathrm{M} L)_{\phi_{i}}$ was done by tracing 100 paths connecting the voxel of interest with equidistant points, sampling the detector. In figure 3 (a) one of such paths is displayed. The ray tracing, using Siddon's algorithm (Siddon 1985), returned the intersection lengths of these rays with the attenuation image voxels. Sensitivity weighting of the intersection lengths with $\cos ^{3} \alpha$, with $\alpha$ the incidence angle, yielded a map of weighted intersection lengths for a certain voxel (figure 3 (b)). Finally, multiplication with the $\mu$ value of the intersected voxel and averaging over all possible paths resulted in $(\mathrm{M} L)_{\phi_{i}}$.

Since our system matrix rotates the image according to the appropriate SPECT angle before applying sensitivity and resolution modeling, we can use the factors $c$ to compensate the rotated image for attenuation. In this way, an approximate attenuation compensation is performed, calculated with every SPECT angle as an average over all spin angles.

2.3.2. Validation GATE was used to validate the correctness of the attenuation values $c$ of our analytic calculation method. Four high count simulations (noise $<0.8 \%$ ) were performed, resulting in four datasets $I_{d}, I_{d, 0}, I_{y}$ and $I_{y, 0}$. For the generation of $I_{d}$ and

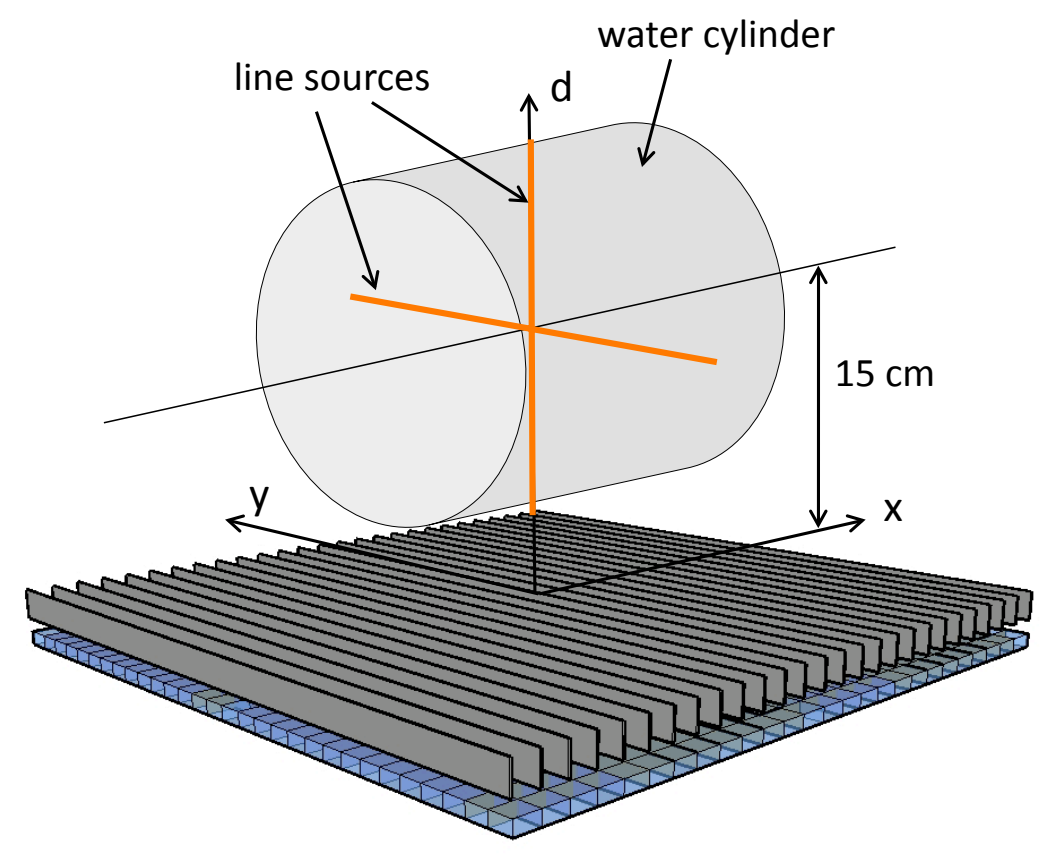

Figure 4. The placement of the line sources with respect to the detector and the water cylinder. 
$I_{y}$, line sources aligned with respectively the $\mathrm{d}$ - and y-axis of the scanner (figure 4) were simulated with a cylindrical water phantom present. The camera was spun over 360 degrees. Binning the recorded data according to the source position resulted in the datasets $I_{d}$ and $I_{y} . I_{d, 0}$ and $I_{y, 0}$ were generated in a similar way with the only difference being the absence of the water cylinder. Since the detector was rotated over all spin angles there exists the following relation between datasets $I$ and $I_{0}$ :

$$
\left.I=I_{0} \frac{1}{\Phi} \sum_{i=0}^{\Phi} \exp \left(-(\mathrm{M} L)_{\phi_{i}}\right)\right)
$$

$\frac{I}{I_{0}}$ should thus be equal to the reciprocal of the attenuation values $c$.

A validation at the level of reconstructed images is also performed by simulating a dataset with and without attenuating medium. The XCAT software phantom (Segars et al 2010) was used to simulate a clinically realistic measurement of a patient injected with Tc-99m-MIBI, used for the assessment of heart perfusion and/or viability. For the purpose of investigating the attenuation correction, the XCAT simulation was performed, once without and once with the XCAT attenuation map presents. The images were then reconstructed first without and then with attenuation correction. The reconstruction of the simulated dataset without attenuation present served as the gold standard image and the reconstructed dataset with attenuation is reconstructed without and with attenuation correction. Both of these latter reconstructions are then compared to the gold standard. For reference, this study was performed with both PH and RS collimation. More details on the XCAT simulation can be found in section 2.6 while image reconstruction is detailed in the next section (2.4).

\subsection{Image reconstruction}

Image reconstruction of the $\mathrm{PH}$ data was performed with plain MLEM, including attenuation correction as proposed by Zeng et al. (Zeng et al 1991). Scatter correction is performed by adding $g_{E S T}$ to the forward projection at each MLEM iteration. For the purpose of reconstruction of the plane integral data collected by the RS collimator, we used a previously proposed split-matrix method for accelerated image reconstruction (Van Holen et al 2007), (Zeng and Gagnon 2004). This method does not model the process to go from plane integrals to image in one single step, but splits the process in two separate steps. The process of plane integration is thus split in two subsequent line integrations. The first line integration to go from sinograms to plane integral data is modeled by $\mathbf{A}$ and the second (matrix $\mathbf{B}$ ) models the step to go from image to sinograms. The resulting system matrix AB is used in an MLEM algorithm:

$$
\hat{f}_{k}^{t+1}=\frac{\hat{f}_{k}^{t}}{\sum_{i} B_{k i} \sum_{j} A_{i j}} \sum_{i} B_{k i} \sum_{j} A_{i j} \frac{g_{j}}{\sum_{i} A_{i j} \sum_{k} B_{k i} \hat{f}_{k}^{t}+g_{E S T}} .
$$

The following iterative process is followed by this algorithm: in a first step, an initial image estimate $\hat{f}_{k}^{t}$ is forward projected with system matrix $\mathbf{B}$ to a sinogram estimate. Next, this sinogram estimate is forward projected using A. For the purpose of scatter 
correction, $g_{E S T}$ is added to the forward projection. This final forward projection is compared to the plane integral data $g_{j}$. The resulting plane integral update is projected backward using $\mathbf{A}^{t}$ and immediately projected backward with $\mathbf{B}^{t}$ to image space where it serves as an update image. After updating and normalizing the original image estimate, the next iteration can start.

In the first part of the system matrix (B), which transforms image space to sinogram space, we model the depth dependent sensitivity as the mean sensitivity in a plane parallel to the detector. Furthermore, depth dependent resolution is also modeled in B. Attenuation compensation is also applied at this level. System matrix A involves a mapping from sinogram to plane integral space. At this level, we do not include any sensitivity modeling nor resolution modeling. No regularisation, inter-update filtering or post-filtering was applied in any of the reconstructions.

\subsection{Monte Carlo simulations of realistic acquisitions}

2.5.1. Image quality phantom For the purpose of a contrast-to-noise comparison, the image quality phantom (Standard Jaszczak Phantom ${ }^{T M}$ ), shown in figure 2 is simulated containing 4 hot spheres (diameters: $9.9 \mathrm{~mm}, 12.4 \mathrm{~mm}, 15.4 \mathrm{~mm}, 19.8 \mathrm{~mm}$ ) and two cold spheres (diameters: $24.8 \mathrm{~mm}$ and $31.3 \mathrm{~mm}$ ). The activity concentration is set in order to have a sphere-to-background activity ratio of 8:1 in the hot spheres. The total activity in the phantom was $370 \mathrm{Mbq}$. The phantom is simulated to be filled with water with an attenuation coefficient $\mu$ of $0.154 \mathrm{~cm}^{-1}$ at $140 \mathrm{keV}$. For the purpose of noise calculation, ten realizations of each dataset were simulated. The rotation radius of the detector was $15 \mathrm{~cm}$ and the acquisition time was set to 8 minutes.

2.5.2. Influence of system modeling The data generated by the Monte Carlo simulation resulted in a one dataset for RS and one for PH. For image reconstruction, we use the algorithm of equation 4 . The data are all reconstructed with attenuation correction as explained in section 2.4. In order to investigate the influence of resolution and sensitivity modeling in the system matrix, the two datasets are reconstructed in three different ways: (i) without any modeling, thus using a simple line integral model (no model); (ii) with resolution modeling (RM) and (iii) both with resolution and sensitivity modeling (RSM). Since for a PH collimator the sensitivity is constant over the FOV, reconstruction RM and RSM will produce equal results. Image quality is then investigated on the basis of contrast recovery and noise. The hot- and cold spot contrast recovery are defined as (Kessler et al 1984):

$$
C R C(\%)=\frac{\frac{m_{l}-m_{b}}{m_{b}}}{C-1} \times 100
$$

with $C$ being the real contrast in the phantom, in our case $C=8$ for the hot spots and $C=0$ for the cold spots. $m_{l}$ and $m_{b}$ are the mean lesion and background activity, averaged over ten realizations. The noise coefficient $(\%)$ is calculated as

$$
N C(\%)=\frac{\sigma_{p}}{m_{p}} \times 100 .
$$


Here, $\sigma_{p}$ and $m_{p}$ respectively are the pixel standard deviation and pixel mean throughout ten realizations of the simulation, averaged over all pixels in a background region of interest.

We evaluated the cold and hot lesion contrast recovery, $\mathrm{CRC}_{c}$ and $\mathrm{CRC}_{h}$, averaged over respectively the two cold and the four hot lesions at a $\mathrm{NC}$ of $25 \%$.

2.5.3. Image quality improvement After investigating the influence of using an accurate system model orthogonal slices through the 3D images, reconstructed with the RM method, will be shown for both the PH and RS collimator at equal NC. Also, 1D profiles through the lesions will be drawn. Next, the contrast-to-noise was plotted for all lesions to investigate the contrast-to-noise properties. This will especially be interesting to study the convergence properties. For the $\mathrm{PH}$ acquisition, the same simulation as described before is repeated in order to study the difference in imaging time to reach equal contrast-to-noise behavior for both collimators.

\subsection{Simulation of a realistic Tc99m-MIBI scan}

The XCAT software was used to simulate a measurement of a patient injected with Tc99m-MIBI. Realistic organ uptake values which have been published before have been used (Jaszczak et al 1996). Background, lungs, liver and heart-wall respectively were assigned activity concentrations of $0.0075 \mathrm{MBq} / \mathrm{ml}, 0.0048 \mathrm{MBq} / \mathrm{ml}, 0.07 \mathrm{MBq} / \mathrm{ml}$ and $0.15 \mathrm{MBq} / \mathrm{ml}$. One defect $(2.8 \mathrm{ml})$ was simulated in the left ventricular anterolateral wall with an activity concentration of $0.0075 \mathrm{MBq} / \mathrm{ml}$. Total activity in the XCAT phantom was $49 \mathrm{MBq}$. The PH and RS scanners were modeled as described in section 2.1, with the only difference of rotation radius $(23 \mathrm{~cm})$ and acquisition time ( 810 seconds). The data were binned for $\mathrm{PH}$ in $96 \times 96$ detector bins for 90 SPECT bins while the RS data were binned in 96 detector bins, 90 SPECT and 90 spin angle bins. The rotation radius was $23 \mathrm{~cm}$. Since the lesion is simulated, we know the exact location of where it should be in the reconstructed images. Therefore, the simulated lesion location is chosen as the lesion ROI $\left(R O I_{l}\right)$. A reference region of interest is chosen in the anteroseptal wall in order to calculate the CRC. Noise is calculated in a background region of interest in the lungs.

\section{Results}

\subsection{Scatter Estimation}

In figure 5 the spectra for the central regions in the small (figure 5 (a) and (b)) and large cylinder (figure 5 (c) and (d)) are shown together with the spectra obtained for the peripheral region in the large cylinder (figure 5 (e) and (f)) for both the PH and RS collimator. A study of these spectra shows that the spectral distribution of scattered photons for the RS collimator are comparable to the $\mathrm{PH}$ collimated scatter distribution. A comparison of the estimated scatter fraction to the true scatter fraction in table 1 


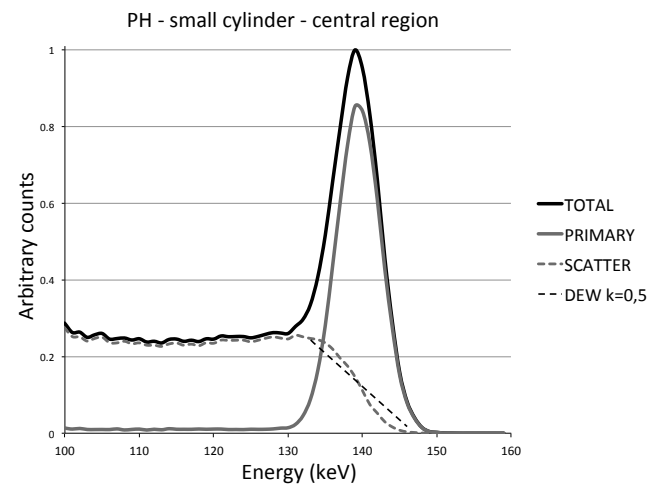

(a)

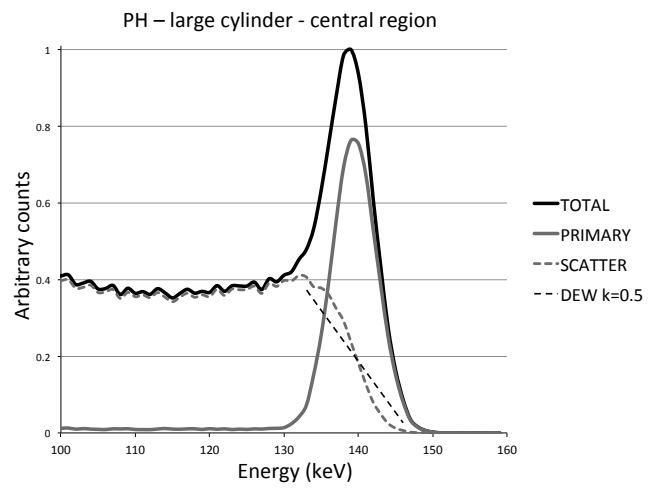

(c)

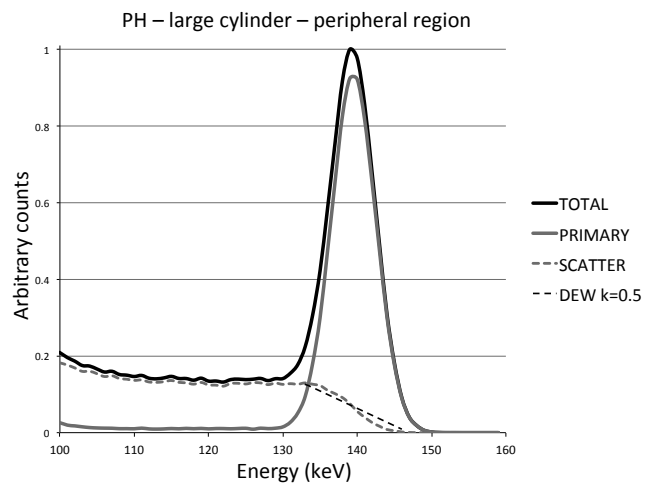

(e)

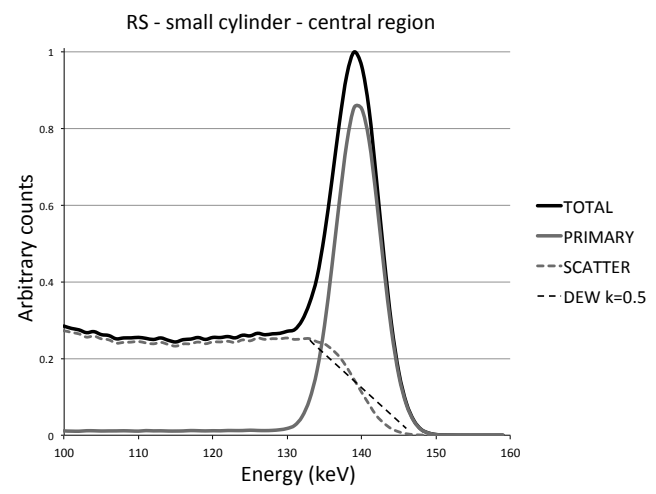

(b)

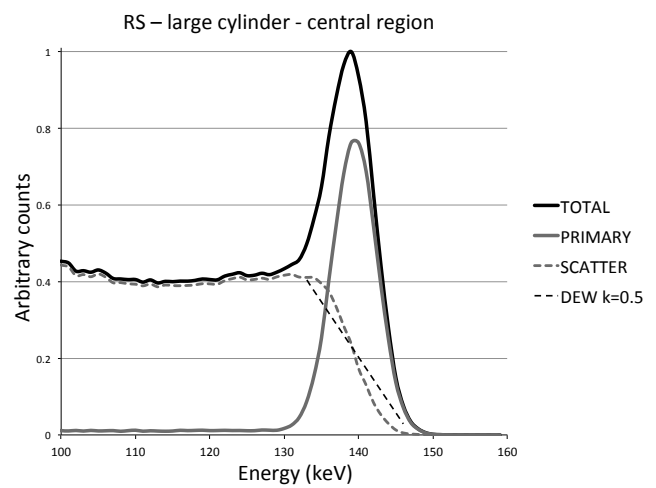

(d)

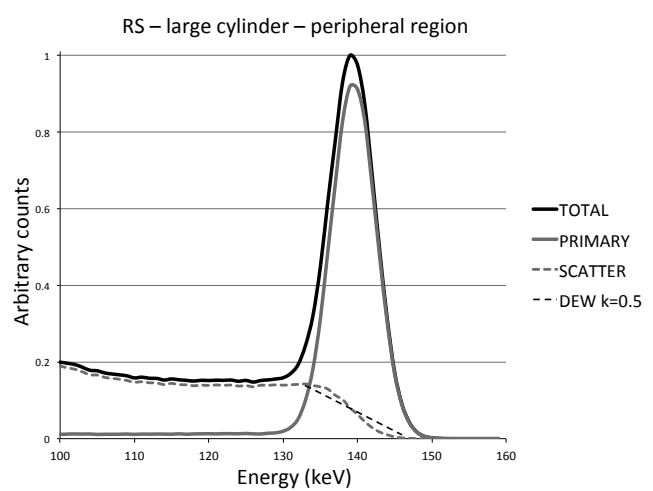

(f)

Figure 5. Energy spectra for investigation of the scatter estimation. In (a) and (b) the spectra from the central region in the small cylinder are shown for the $\mathrm{PH}$ and RS collimation respectively. In (c) and (d) the spectra from the central region in the large cylinder are shown and in (e) and (f) the spectra from the peripheral region in the large cylinder are shown for the respective types of collimation. 
learns that the error made by the DEW scatter estimation technique makes small errors which are comparable for $\mathrm{PH}$ and RS.

\begin{tabular}{lccccc}
\hline & \multicolumn{2}{c}{$\mathrm{PH}$} & & \multicolumn{2}{c}{ RS } \\
\cline { 2 - 3 } \cline { 5 - 6 } & True & Estimated & & True & Estimated \\
\hline \hline small central & 21.8 & 22.6 & & 21.9 & 22.7 \\
large central & 33.4 & 32.7 & & 33.8 & 35.0 \\
large peripheral & 11.7 & 12.2 & & 12.8 & 13.1 \\
\hline \hline
\end{tabular}

Table 1. Comparison of ectimated and true scatter fractions (\%)

\subsection{Attenuation correction}

The calculations of $c$ were based on an attenuation map derived from the cylindrical phantom. The results are summarized as a line profile through the reciprocal of the calculated attenuation values $c$ (ANALYTIC) for varying $d(y=x=0)$ and for varying y $(d=190 \mathrm{~mm}$ and $x=0)$ in figure 6 (a) and figure 6 (b) respectively. The simulation results (GATE) in figures 6 (a) and (b) are the ratio of respectively $I_{d}$ and $I_{d, 0}$ and $I_{y}$ and $I_{y, 0}$. These profiles show very good agreement and suggest the proposed analytic calculation method for the attenuation values is suitable for attenuation compensation during our iterative reconstruction. The difference between both profiles is also plotted (delta) and remains smaller than 1\%. These errors are believed to be due to discretization errors in the calculation method. A comparison

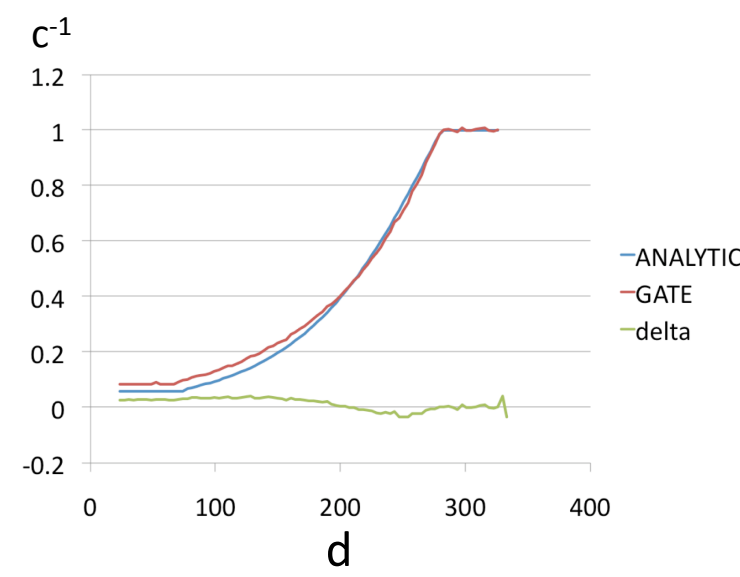

(a)

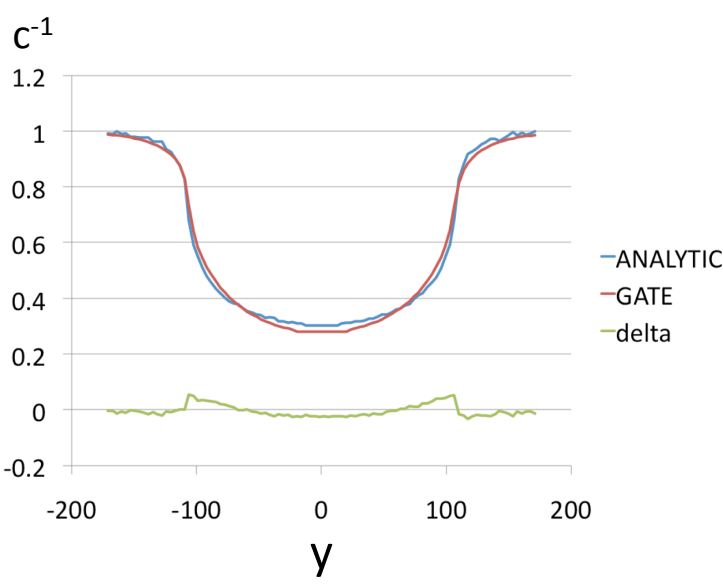

(b)

Figure 6. Plot of simulation-based (GATE), calculation-based (ANALYTIC) values for attenuation correction, together with their difference (delta). (a) shows the values along $\mathrm{d}$ for $y=x=0$ and (b) shows the values along y for $x=0$ and $d=19 \mathrm{~cm}$.

at the level of reconstructed XCAT images is made in figure 7. Coronal slices through the reconstructed XCAT acquisitions at the position of the heart lesion are shown in 


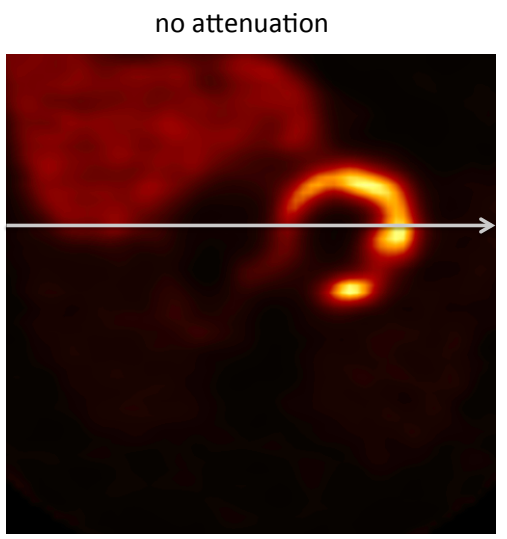

(a)

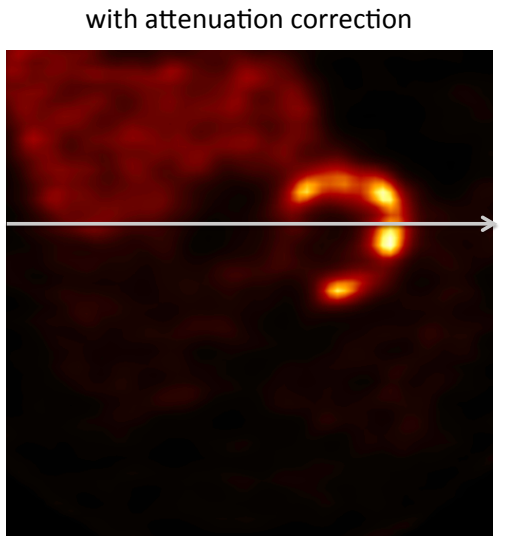

(c)

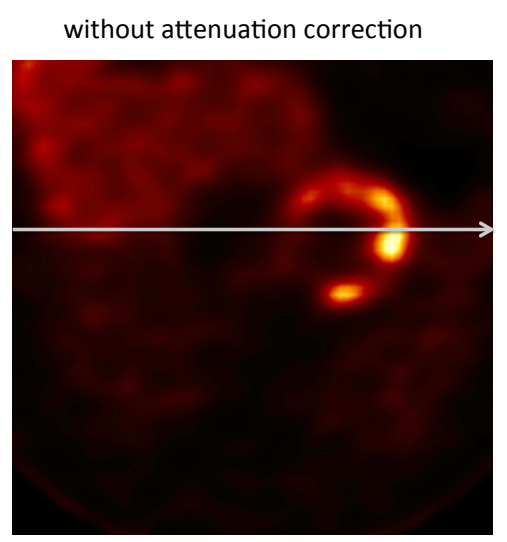

(b)

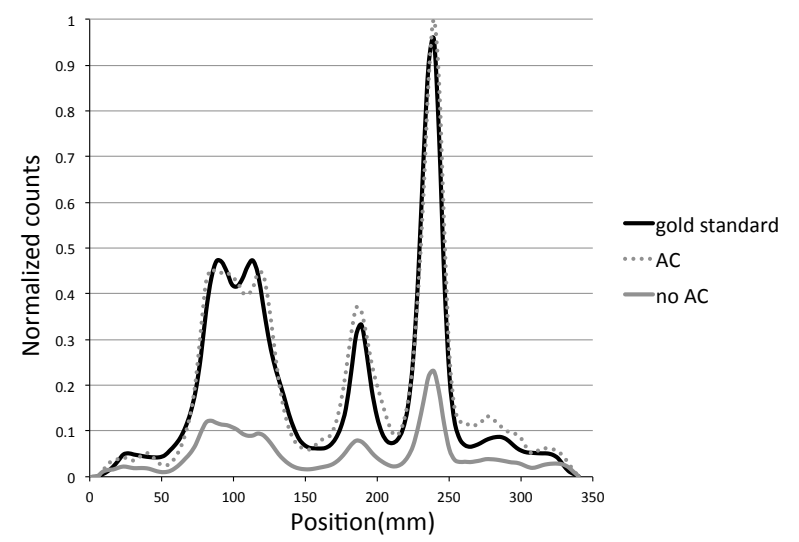

(d)

Figure 7. Coronal slices through reconstruction of the XCAT phantom at the position of the heart lesion. In (a) no attenuation was simulated while in (b) attenuation was simulated but no attenuation correction was used during reconstruction. In (c) attenuation was simulated and corrected during reconstruction. In (d), a profile through these images shows the effect of attenuation correction.

figure 7(a),(b) and (c), respectively for the case where no attenuation was simulated, the case where no attenuation correction was applied and the case where attenuation correction was applied during reconstruction. From the profiles, plotted in figure figure $7(\mathrm{~d})$, it can be appreciated that the attenuation correction restores the absolute image values to the correct count level. The reconstructed images in figure 7(b) and (c) show more noise compared to figure 7(a) because of the lower number of measured counts due to attenuation. Compared to figure $7(\mathrm{~b})$ where no attenuation correction is applied, figure 7 (c) not only restores the absolute count level, but also restores the relative voxel intensity. It can be clearly seen that the inner heart wall's count level is restored to the original level of figure 7 (a). Also, the liver uptake in figure 7 (c) is more uniform compared to figure 7 (b). 


\subsection{Image quality phantom}

3.3.1. Influence of system modeling The influence of system modeling is evaluated at $25 \%$ NC. To reach this level of noise, different iteration numbers had to be used for the different reconstruction methods. They are indicated in table 2 . In the same table, the results of the $\mathrm{CRC}_{c}$ and $\mathrm{CRC}_{h}$ are summarized for the $\mathrm{PH}$ and $\mathrm{RS}$ collimated acquisitions. For the $\mathrm{PH}$ collimator, an average increase of about $10 \%$ with respect to the case without system resolution modeling is found for both hot and cold contrast recovery when applying a depth dependent resolution model. It can be seen that for the small hot lesions the increase is lower compared to the large hot lesions.

\begin{tabular}{lccccccc}
\hline & \multicolumn{2}{c}{ PH } & & \multicolumn{3}{c}{ RS } \\
\cline { 2 - 3 } \cline { 6 - 8 } \cline { 6 - 8 } & wo model & RM & & wo model & RM & RSM \\
\hline \hline number of iterations & 8 & 16 & & 640 & 1040 & 960 \\
\hline \hline 9.9mm hot lesion & 5.57 & 8.98 & & 12.6 & 15.9 & 15.4 \\
12.4mm hot lesion & 12.6 & 20.9 & & 26.6 & 35.5 & 35.7 \\
15.4mm hot lesion & 19.7 & 32.6 & & 31.1 & 42.4 & 43.0 \\
19.8mm hot lesion & 22.7 & 36.5 & & 39.9 & 48.9 & 49.3 \\
24.8mm cold lesion & 16.3 & 26.0 & & 33.4 & 40.7 & 42.2 \\
31.3mm cold lesion & 22.3 & 33.0 & & 45.7 & 52.1 & 52.6 \\
\hline average hot & 15.1 & 24.8 & & 27.6 & 35.7 & 35.6 \\
average cold & 19.3 & 29.5 & & 39.6 & 46.4 & 47.4 \\
\hline \hline
\end{tabular}

Table 2. Mean cold and hot contrast recovery coefficient at $25 \%$ noise for (a) the PH and for (b) the RS reconstruction. Reconstruction without any modeling ('wo model'), with resolution ('RM') and with additional sensitivity modeling ('RSM') are compared

For the RS collimator, an increase of about $8 \%$ and $7 \%$ is found for respectively hot and cold contrast recovery when applying a depth dependent resolution model. An additional increased $\mathrm{CRC}_{c}$ of $1 \%$ is found when sensitivity is additionally modeled. Hot contrast did not increase due to the sensitivity modeling. The better contrast recovery, found for the RS collimator is due to better contrast-to-noise performance of this collimator. The results of the contrast-to-noise analysis are detailed in the next section.

\subsection{Contrast-to-noise}

In table 2, for the cases of system resolution modeling, an increased $(+10.9 \%)$ hot $\mathrm{CRC}$ can be seen for the RS collimator compared to PH. Also an increase $(+16.9 \%)$ in cold $\mathrm{CRC}$ is found for the $\mathrm{RS}$ compared to the PH collimator. Figure 8 shows the contrast-to-noise plot for the average cold CRC. The cold lesions show better contrast recovery for $\mathrm{RS}$ compared to the $\mathrm{PH}$ collimator. To have an idea about the quantitative improvement, the imaging time was increased for the $\mathrm{PH}$ collimator. The results for a 2, 3 and 4 times longer PH acquisition are also plotted in figure 8. From these plots, it can 


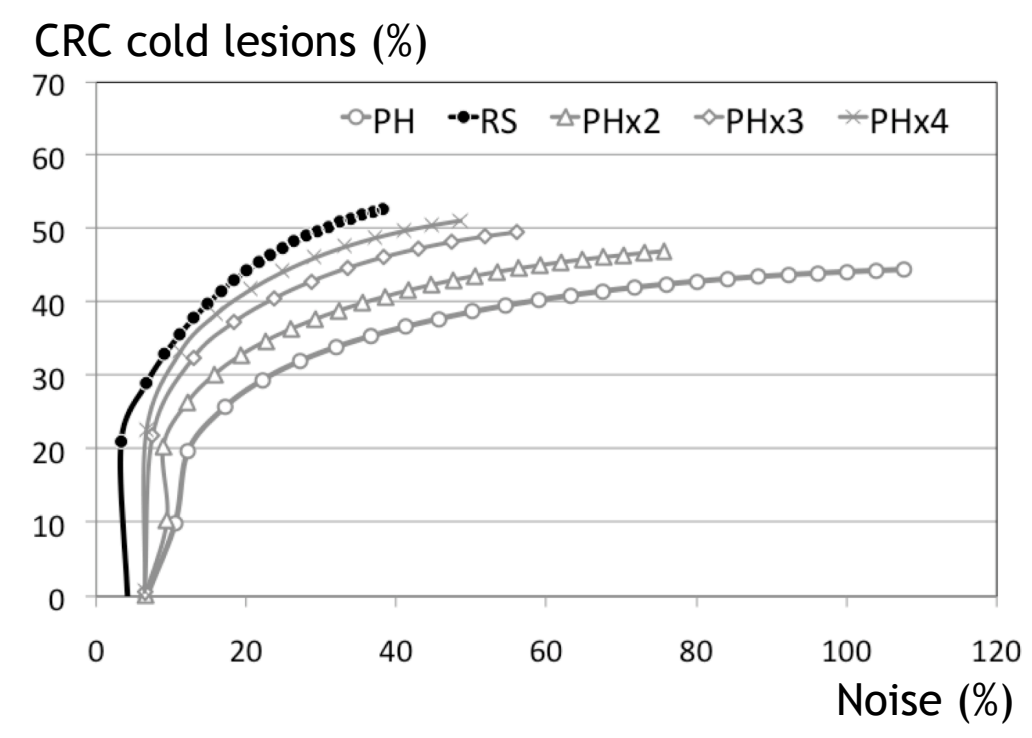

Figure 8. Contrast-to-noise averaged over both cold lesions. Reconstructions performed by the RM method.

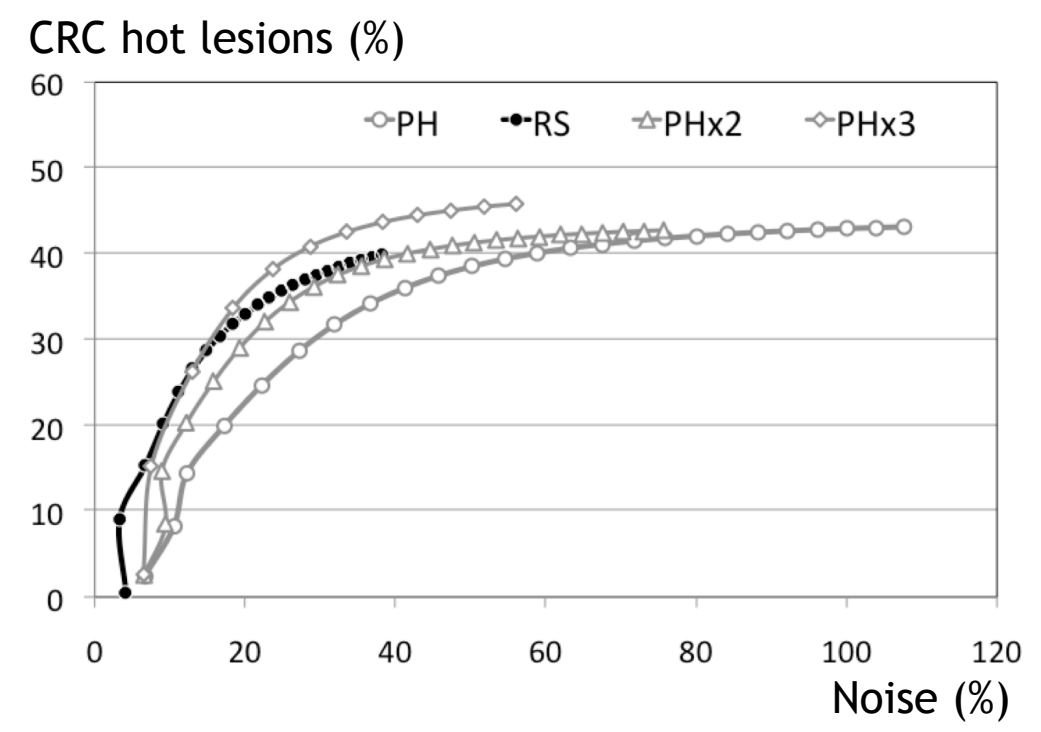

Figure 9. Contrast-to-noise averaged over all four hot lesions. Reconstructions performed by the RM method

be seen that, to obtain the same contrast-to-noise, the $\mathrm{PH}$ collimator needs more than 4 times longer acquisition time compared to the RS collimator. The average result for the hot lesions is shown in figure 9 . This plot shows that the RS collimator needs 2 to 3 times less scan time than a $\mathrm{PH}$ collimator in order to obtain similar contrast-to-noise characteristics. 


\subsection{Tomographic images}

A transverse slice through the images at equal noise (10\%) is shown in figure 10 (a) and in figure 10 (b) for respectively the $\mathrm{PH}$ and the RS collimator. It can be seen from the images already that the RS collimator improves the contrast. The profile drawn through the lesions in figure 11 confirms the better contrast recovery with the RS collimator.

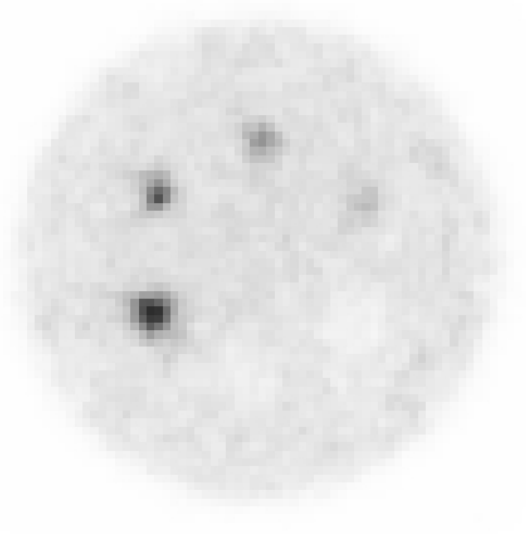

(a)

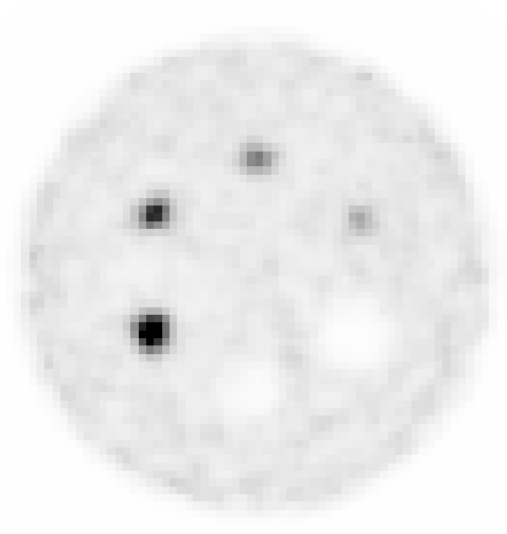

(b)

Figure 10. Reconstructed images from the (a) the PH and (b) from the RS. Noise is $10 \%$.

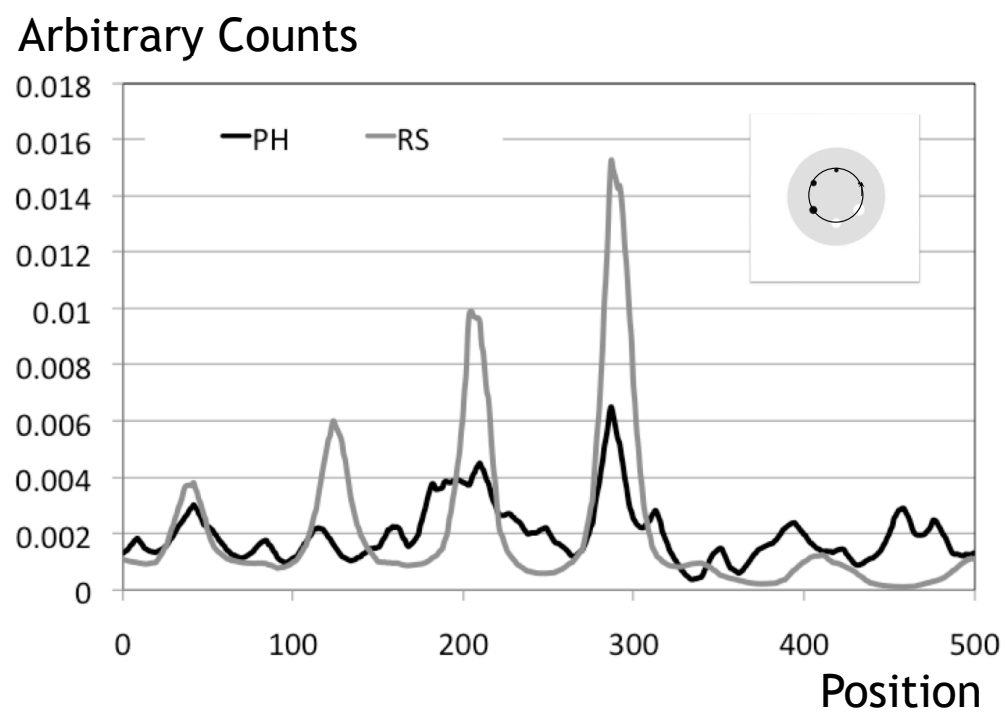

Figure 11. Circular profiles through the lesions are drawn, normalized to the total number of counts under the profile. Noise is $40 \%$. 


\subsection{Tc-99m-MIBI scan with the XCAT phantom}

The total number of counts detected with the RS collimator was found to be 27 times higher compared to the $\mathrm{PH}$ collimator for the XCAT simulations. The contrast-to-noise is calculated for the $2.8 \mathrm{ml}$ heart defect. The ROI of the lesion was exactly aligned with the simulated lesion in the XCAT phantom. Noise was calculated in a ROI in the lungs where the simulated activity was uniform. From figure 12, it can be seen that the contrast-to-noise for the RS collimator is better. At equal contrast, the RS has lower noise and at equal noise, the RS has better contrast. The results are consistent between the case where attenuation was simulated and the case where attenuation was omitted from the simulation. The overall noise level increases when attenuation is simulated due to the lower count level, but again, the RS collimator outperforms the PH collimator.

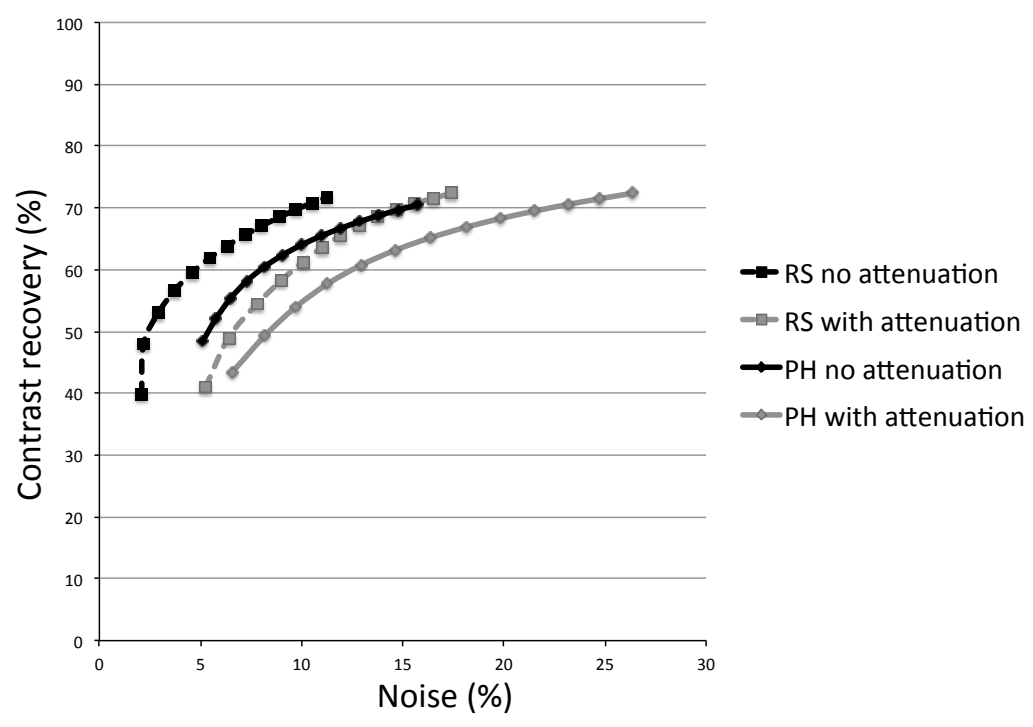

Figure 12. Contrast-to-noise for the $2.8 \mathrm{ml}$ heart lesion for the $\mathrm{PH}$ collimator and the RS collimator with and without presence of surrounding activity.

From the coronal slices through the heart lesion in figure 13, the slightly better image quality for the RS collimator can be appreciated. The images are at $60 \% \mathrm{CRC}$. 


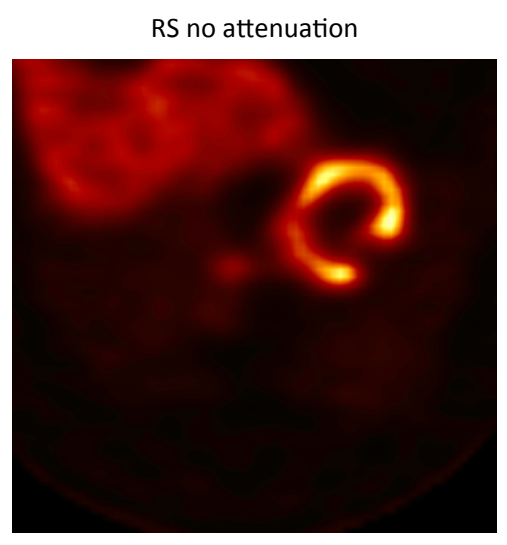

(a)

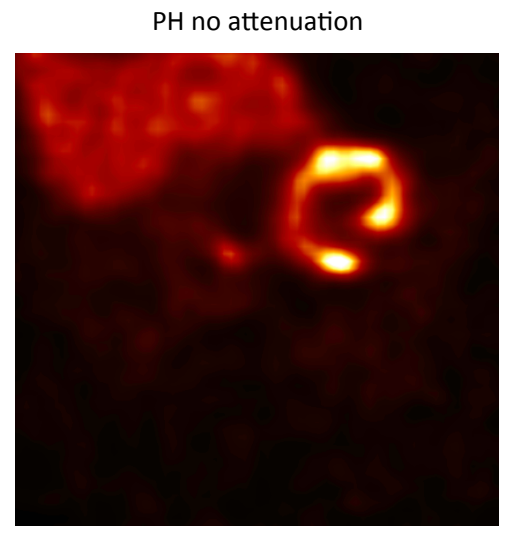

(c)

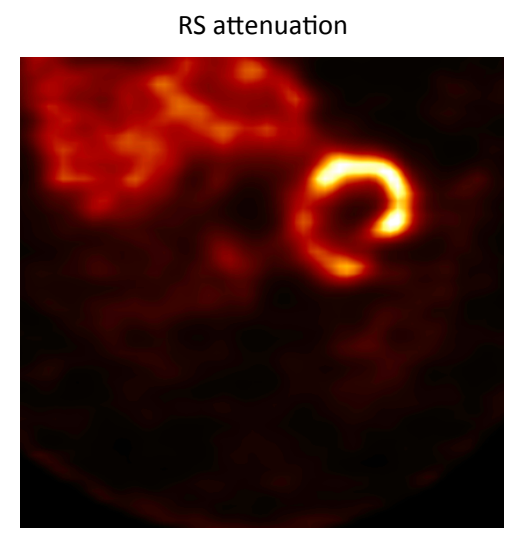

(b)

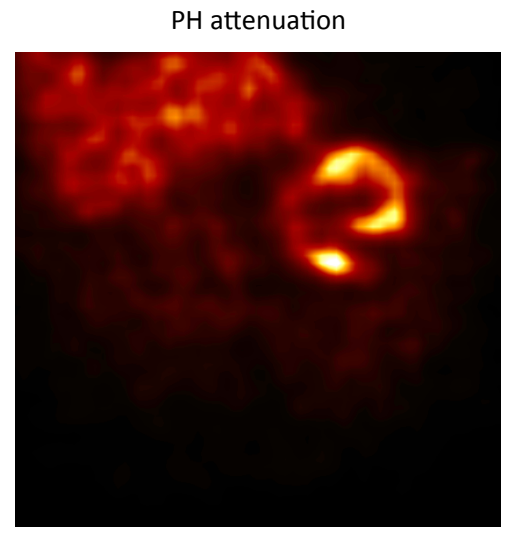

(d)

Figure 13. A coronal slice through the heart defect for (a) the RS collimator without attenuation in the simualtion and (b) with attenuation simulated and corrected during reconstruction. Coronal slice for the $\mathrm{PH}$ collimator (a) without and (b) with attenuation simulated. Images are displayed at equal contrast (60\%).

\section{Discussion}

In this study, dual energy window scatter estimation was used for RS collimated data and was found at least equally accurate compared to DEW scatter correction for PH collimated acquisitions. Furthermore, an analytic method for calculating attenuation factors for every voxel at every SPECT angle was proposed and validated. This enabled us to correct for attenuation in a previously proposed reconstruction method for plane integral data. With these tools available, a realistic Monte Carlo simulation of an image quality phantom could be reconstructed. An investigation of the influence of system modeling shows that both a PH and RS substantially gain image quality when a depth dependent resolution model is used during reconstruction. An increased contrast of about 8\%-10\% was found for both collimators at 25\% noise. An additional model for sensitivity did not significantly increase image quality further for the RS collimator. Also when no modeling is used at all, the RS collimator performs better compared to the 
PH collimator (20\% higher cold contrast and $12 \%$ higher contrast at $25 \%$ noise). These findings are consistent with the findings of Wang (Wang et al 2004). The improvements found by Lodge (Lodge et al 1996), where FBP was used as a reconstruction algorithm, were more modest. Therefore, we believe that it is not the modeling, but the nature of MLEM, which includes a statistical model of the data, that favors the RS collimation. While in FBP, all noisy projections are simply backprojected and added in image space for every spin and every SPECT angle, MLEM tries to find a maximum likelihood solution by iteratively correcting an initial estimate with a smooth correction term.

The contrast-to-noise investigation shows that SPECT images obtained with a rotating slat collimator in combination with iterative reconstruction and accurate modeling provide a better contrast-to-noise trade-off compared to images obtained with an equivalent $\mathrm{PH}$ collimator. In terms of imaging time, equal average cold contrast-tonoise is found in a more than 4 times shorter scan time for the RS collimated detector. For the contrast-to-noise, averaged over the hot lesions, the results show a 2 to 3 times improvement in terms of acquisition time. This study thus not only shows better hot spot contrast recovery, which was also found by Lodge (Lodge et al 1996), but also proves better cold spot contrast recovery, which is consistent with the results obtained by Wang (Wang et al 2004). These results were obtained however with a slat collimated solid-state strip detector, but in that study, no depth dependent resolution was modeled and no attenuation compensation was used. Also, it was unclear whether the improved contrast-to-noise was due to the better solid state detector or due to the slat collimator. This study indicates the improvement is due to the collimator. We need to note however that an effective sensitivity gain could be lower when more detectors are used or when smaller rotation radii are possible. Due to the extra rotation needed by the RS design, more space is needed compared to a $\mathrm{PH}$ system with equal size detectors not to let the detectors run in to each other while spinning.

The case study of the Tc-99m-MIBI scan with the XCAT phantom shows that in a clinical setting, where a larger scan radius has to be used and where there is more activity throughout the FOV, the RS collimator still reaches better image quality.

\section{Conclusion}

In this paper, an attenuation correction method for the reconstruction of plane integral data was developed in order to study the tomographic image quality with respect to a parallel hole collimator. Furthermore, a standard scatter correction method was validated. For a standard image quality phantom, better image quality was obtained for both hot and cold lesions. In the clinically realistic case of a Tc-99m-MIBI scan of a heart defect, the rotating slat collimator also obtains better images. Therefore, tomographic rotating slat is an interesting alternative for existing collimation. 


\section{Acknowledgments}

Research funded by a the Fund for Scientific Research Flanders (FWO, Belgium) and by the Ghent University.

\section{References}

Accorsi R 2006 High-efficiency, high-resolution SPECT techniques for cardiac imaging Proceedings of Science.

Chang L 1978 A method for attenuation correction in radionuclide computed tomography IEEE Transactions on Nuclear Science $\mathbf{2 5} 638-643$.

Entine G, Luthmann R, Mauderli W, Fitzgerald L, Williams C and Tosswill C 1979 Cadmium Telluride gamma camera IEEE Transactions on Nuclear Science 26 552-558.

Gagnon D, Zeng G, Links M, Griesmer J and Valentino F 2001 in 'Nuclear Science Symposium Conference Record' Vol. 2 pp. 1156-1160.

Griesmer J, Kline B, Grosholz J, Parnham K and Gagnon D 2001 in 'Nuclear Science Symposium Conference Record' Vol. 2 pp. 1050-1054.

Jaszczak R, Gilland D, McConnick J, Scarfone C and Coleman R 1996 The effect of truncation reduction in fan beam transmission for attenuation correction of cardiac SPECT IEEE Transactions on Nuclear Science 43 2255-2262.

Kessler R, Ellis J and Eden M 1984 Analysis of tomographic scan data: limitations imposed by resolution and background Journal of Computer Assisted Tomography 8 514-22.

Keyes W 1975 Correspondence: The fan-beam gamma camera Physics in Medicine and Biology 20 489493.

Koral K, Swailem F, Buchbinder S, Clinthorne N, Rogers W and Tsui B 1990 SPECT dual-energywindow Compton correction: scatter multiplier required for quantification. Journal of Nuclear Medicine 31 90-98.

Lodge M, Binnie D, Flower M and Webb S 1995 The experimental evaluation of a prototype rotating slat collimator for planar gamma camera imaging Physics in Medicine and Biology 40 427-448.

Lodge M, Webb S, Flower M and Binnie D 1996 A prototype rotating slat collimator for single photon emission computed tomography IEEE Transactions on Medical Imaging 15 500-511.

Moore S, Kouris K and Cullum I 1992 Collimator design for single photon emission tomography European Journal of Nuclear Medicine 19 138-150.

Santin G, Strul D, Lazaro D, Simon L, Krieguer M, Vieira Martins M, Breton V and Morel C 2003 GATE, a Geant4-based simulation platform for PET and SPECT integrating movement and time management IEEE Transactions on Nuclear Science 50 1516-1521.

Segars W, Sturgeon G, Mendonca S, Grimes J and Tsui B 2010 4D XCAT phantom for multimodality imaging research Medical Physics 37 4902-4915.

Siddon R 1985 Fast calculation of the exact radiological path for a three-dimensional CT array. Medical Physics 12 252-255.

Tosswill C 1977 Computerized rotating laminar collimation imaging system US patent application 646 (granted December 1977) pp. 917-967.

Van Holen R, Vandenberghe S, Staelens S and Lemahieu I 2007 Fast 3D image reconstruction for rotating slat collimated gamma cameras. Proceedings 9th International Meeting on Fully ThreeDimensional Image Reconstruction in Radiology and Nuclear Medicine pp. 390-392.

Van Holen R, Vandenberghe S, Staelens S and Lemahieu I 2008 Comparing planar image quality of rotating slat and parallel hole collimation: influence of system modeling Physics in Medicine and Biology 53 1989-2002.

Vandenberghe S, Staelens S, Byrne C L, Soares E J, Lemahieu I and Glick S J 2006 Reconstruction of 
$2 \mathrm{~d}$ pet data with monte carlo generated system matrix for generalized natural pixels. Physics in Medicine and Biology 51 3105-3125.

Wang W, Hawkins W and Gagnon D 2004 3D RBI-EM reconstruction with spherically-symmetric basis function for SPECT rotating slat collimator Physics in Medicine and Biology 49 2273-2292.

Webb S, Binnie D, Flower M and Ott R 1992 Monte Carlo modeling of the performace of a rotating slit collimator for improved planar gamma camera imaging Physics in Medicine and Biology 37 1095-1108.

Wieczorek H and Goedicke A 2006 Analytical model for SPECT detector concepts IEEE Transactions on Nuclear Science $\mathbf{5 3}$ 1102-1112.

Zeng G, Gagnon D, Natterer F, Wang W, Wrinkler M and Hawkins W 2003 Local tomography property of residual minimization reconstruction with planar integral data IEEE Transactions on Nuclear Science 50.

Zeng G, Gullberg G, Tsui B and Terry J 1991 Three-dimensional iterative reconstruction algorithms with attenuation and geometric point response correction IEEE Transactions on Nuclear Science 38 693-702.

Zeng G L and Gagnon D 2004 Image reconstruction algorithm for a SPECT system with a convergent rotating slat collimator IEEE Transactions on Nuclear Science 51 142-148.

Zhou L, Defrise M, Vunckx K and Nuyts J 2010 Comparison between parallel hole and rotating slat collimation: Analytical noise propagation models IEEE Transactions on Medical Imaging $292038-2052$. 